\title{
Planets, evolved stars, and how they might influence each other.
}

\author{
Eva Villaver \\ Departamento de Física Teórica, Universidad Autónoma de Madrid \\ Facultad de Ciencias, 28049 Madrid, Spain \\ email: eva.villaver@uam.es
}

\begin{abstract}
Over the last 20 years planetary searches have revealed a wealth of systems orbiting stars on the main sequence. Most of these low-mass stars eventually will evolve into the Giant phases before entering the planetary nebulae $(\mathrm{PNe})$ stage. In the last years, the presence of planets has also been discovered around more massive evolved stars, mostly, along the Red Giant but also along the Horizontal Branch. Moreover, disks have been found around White Dwarfs presumably formed by tidally disrupted asteroids. In all, there is evidence that an evolved (ing) star might influence the survival of planets. In this review I will try to summarize such evidence but furthermore I will present the other side of the story, that is, how the presence of a planet might alter the evolution of stars and with that the PN formation.
\end{abstract}

Keywords. Stars: AGB and post-AGB, planets and satellites: general, planetary nebulae: general.

\section{Introduction}

The focus of this paper is on low-and intermediate-mass stars, those in the mass range between 1 and $5-8 \mathrm{M}_{\odot}$ (the upper mass limit is not well defined) that after losing most of their mass in the Asymptotic Giant Branch (AGB) phase, end their lives as white dwarfs with masses that peak at $\sim 0.6 \mathrm{M}_{\odot}$. These stars are the progenitors of Planetary Nebulae (PNe), and therefore, represent the evolutionary endpoints of most of the exoplanets that have been discovered orbiting main sequence stars.

The large majority of the extrasolar planets known so far, are found orbiting main sequence stars with masses similar to the mass of the Sun. Stellar evolution theory predicts, that once the hydrogen is exhausted as the nuclear fuel in the stellar core, the star will experience dramatic changes capable of determining the fate of any planet that might be orbiting it.

Low-and intermediate-mass stars evolve into two giant phases, the Red Giant Branch and the AGB phase during which the radius of the star increases orders of magnitude. The star reaches its maximum radius along the AGB. It is also at this time when the star loses most of its mass. After this, the ionization of the nebula takes place as the stellar remnant reaches the highest temperature in its evolution, at the constant luminosity defined by the mass reached at the end of the AGB (see e.g. Villaver et al. 2002ab). Finally, the core will contract following the white dwarf cooling track as the nebula gets diluted.

The expected fate of planets currently orbiting main sequence stars relies heavily on our knowledge of stellar evolution. In this paper, we will try to summarize theoretically how an evolved star is expected to influence the survival of planets. But furthermore, we will show how in which ways the presence of a planet might alter the evolution of stars and with that the Planetary Nebulae formation. 

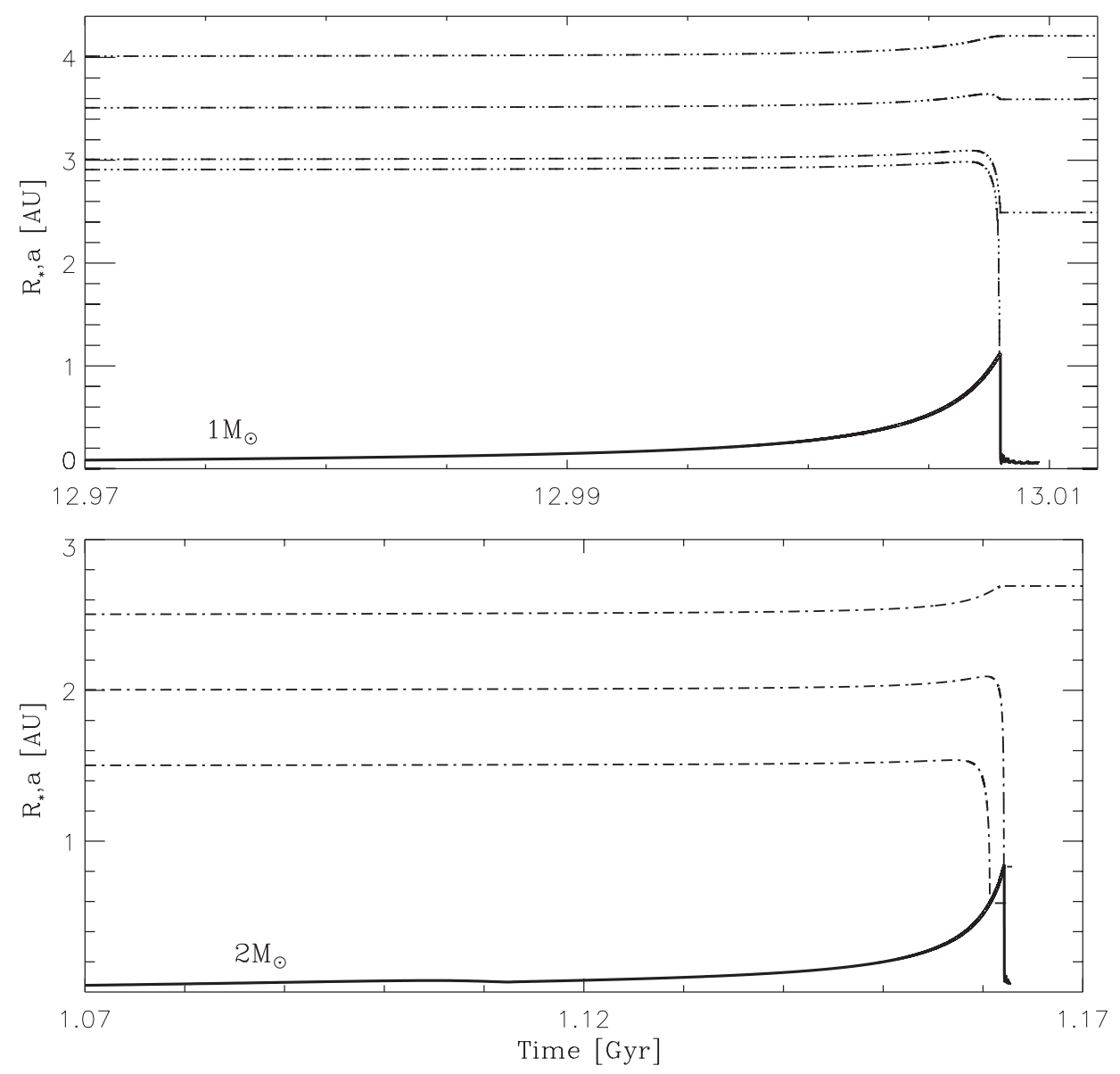

Figure 1. Evolution of the orbital separation of a planet with $M_{\mathrm{p}}=M_{\mathrm{J}}$ (dash-dotted line) and the radius of the star (solid line) along the RGB. The panels represent the stellar MS masses considered ( 1 and $2 \mathrm{M}_{\odot}$ top and bottom panels respectively) and are marked at the bottom left corner of each plot. The different dashed lines are the orbits for different initial orbital distances.

\section{The giant phases}

Very recently, the search for planets has been extended to stars that have evolved off the main sequence into the giant stage (see e.g. Johnson et al. 2007; Lovis \& Major 2007; Wright et al. 2009). One can learn a lot from these searches regarding the planet formation mechanism around more massive stars, as they allow to extend the mass range of applicability of the Doppler techniques. Spectral types earlier than late-F on the main sequence have large rotational velocities and very few spectral lines. However, once the star leaves the main sequence and the radius increases, conservation of angular ensures that the rotational velocities decrease.

One of the most important trends found by these surveys is the lack of close-in planets orbiting stars with masses $M \geqslant 1.5 \mathrm{M}_{\odot}$, with only one exception (Johnson et al. 2010), despite the fact that close-in or hot jupiters are found around $\approx 20 \%$ of main sequence stars with $M<1.2 \mathrm{M}_{\odot}$.

Given that the stars are more massive than their main sequence analogues, one of the first question that arises is try to understand if this difference is primordial, i.e. a consequence of the planet formation mechanism around more massive stars. Along these 
lines, it has been shown by Currie (2009) that the dependence of the lifetime of the gaseous disk on the stellar mass could result in halting the inward migration of planets around high-mass stars, thus explaining the observed lack of short period planets around these stars. However, before primordial mechanisms are involved, stellar evolution has to be ruled out, given that we know that the planet hosting stars are giant and subgiants evolutionary phases.

In Villaver \& Livio (2009), we try to determine whether stellar evolution could explain the observed distribution of the semi-major axes of planetary orbits around evolved stars (i.e., semi-major axis $>0.5 \mathrm{AU}$ ). Conservation of angular momentum gives the equation for the rate of change in the orbital radius of the planet along the Red Giant Branch. The stellar evolution timescales (in the absence of significant mass loss) are set by the rate of consumption of the nuclear fuel. Since the nuclear burning has been exhausted in the core, during the RGB hydrogen burning continues in a shell outside the helium core, which now, devoid of energy sources, is contracting and heating up. As the core contracts the envelope expands and cools. The stellar effective temperature decreases while the star's radius and luminosity increase.

Villaver \& Livio (2009) computed the orbital evolution for a range of initial orbital distances and planet and stellar masses in order to determine the minimum initial orbital distance for which a planet will avoid being tidally captured by the expanding star $\left(R_{*}^{\max }\right)$. For illustration, some of these orbits (dash-dotted lines) are plotted in Fig. 1, where we have used a planet with a mass of $M_{\mathrm{p}}=1 M_{\mathrm{J}}$ orbiting stars with different main sequence masses to the end of the red giant branch phase. The evolution of the stellar radius is shown as a solid line.

Figure 1 illustrates the three possible outcomes of orbital evolution:

- Beyond a certain initial orbital separation, the orbital separation simply increases, due to systemic mass loss.

- There is a range of initial orbital separations for which the orbit decays, but the planet avoids being engulfed.

- Inward from some critical, initial orbital separation, the planet is engulfed mostly due to tidal interaction.

For all the initial orbits that satisfy the condition $a_{\mathrm{o}} \leqslant R_{*}^{\max }$ the planet gets engulfed by the star at same point before the end of the RGB phase. The more massive the planet the stronger is the tidal interaction with the star, and therefore the sooner the orbit decays to meet the stellar radius (see Villaver \& Livio 2009).

\section{Planet-star interactions}

If the planet's initial orbit is within the reach of the stellar radius, the planet will get inside the stellar envelope, and it is at this point that the presence of a planet might influence the evolution of the star in several ways. Some of them are outlined below.

\subsection{Rapid $R G B$ rotators}

Rotation is an important physical parameter that could provide evidence of an interaction with a stellar or substellar companion. Reduced rotational speeds are expected in giant stars, because due to angular momentum conservation they should spin down as their radius expands. Cool giants $\left(T \leqslant 5,000 \mathrm{~K}\right.$ ) are characterized by $v \sin i \leqslant 2 \mathrm{~km} \mathrm{~s}^{-1}$ (de Medeiros et al. 1996; Gray 1981).

High velocity rotators are unexpected with normal stellar evolution. But Carlberg et al. (2009) found $2.2 \%$ rapid rotators in a sample of $\approx 1300$ giant stars. Either an internal 

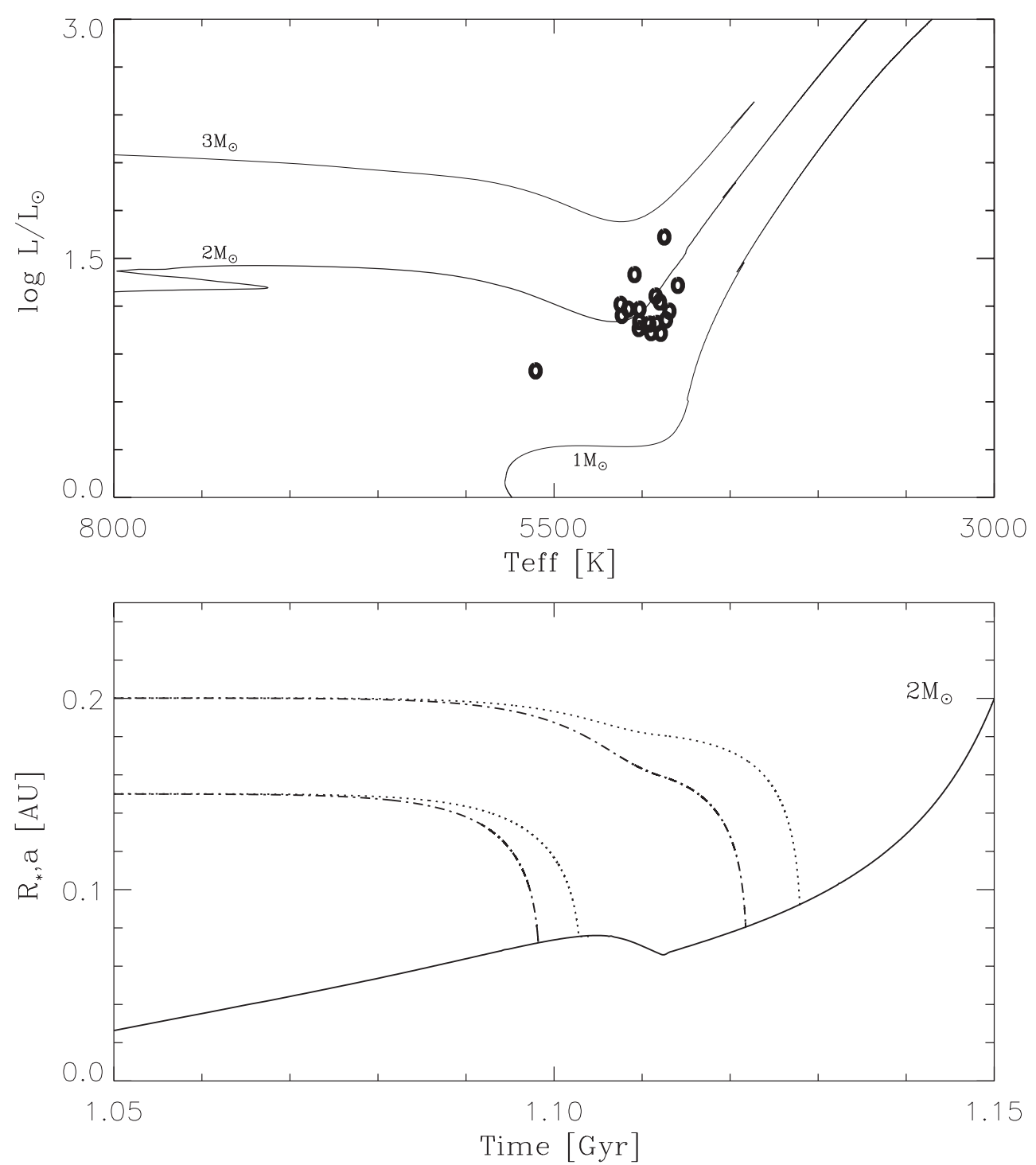

Figure 2. Top: The evolution of 1,2 , and $3 \mathrm{M}_{\odot}$ stars during the red giant branch along the HR diagram. Bottom: The evolution of the orbital separation of a planet with $M_{\mathrm{p}}=3 M_{\mathrm{J}}$ (dotted line) and $M_{\mathrm{p}}=5 M_{\mathrm{J}}$ (dash-dotted line) for two different initial orbital separations. The evolution of the stellar radius along the RGB is also shown as a solid line. Note that the maximum radius reached by the star during the RGB has not been plotted in the bottom panel. The points represent the location of detected stars hosting planets taken from the literature.

or external mechanism has to spun up the star, in the absence of a stellar companion or cluster environment, a planet or brown dwarf offers a viable option.

\subsection{Li-rich giants}

Lithium is easily destroyed within the main sequence star's lifetime. If it survives, it does it in a very thin surface layer that is expected to get diluted by a factor of 60 (see e.g. Iben 1967) as the convective envelope develops when the star evolves into the giant phase.

Unusually large amounts of Lithium have been found in $\approx 1 \% \mathrm{~K}$-giants in the solar neighborhood (Brown et al. 1989). Alexander (1967) was the first to suggest that the 
swallowing a massive planet by a giant star could explain Lithium overabundance in these stars. However, recently Kumar, Reddy \& Lambert (2011) argued that the finite luminosity spread they found in their spectroscopy survey for Li-rich K giants, does not support the idea that $\mathrm{Li}$ enhancement is in general a consequence of swallowing a planet.

\subsection{Common envelope evolution}

CE evolution is a poorly understood phase and important part of the evolution of binary systems (see Izzard contribution in this proceedings). Common envelope is most likely expected to happen during the giant phases of the star. One the planet is inside this common envelope, the binary orbit between the core of the giant and the secondary (a planetary companion in this case) shrinks due to friction and torques leading to either a compact binary or a merger. The energy generated due to orbital shrinkage can heat and eventually lead to envelope ejection. It has been also shown to be capable of generating dynamos in PN progenitors (Nordhaus et al. 2007).

\subsection{Enhanced mass-loss}

A massive orbiting companion can transfer angular momentum to the stellar envelope and enhance its mass-loss. Livio \& Soker (2002) estimated that for the companion to have significant effect on the stellar mass-loss it must increase the angular momentum of the envelope by an order of magnitude.

Nordhaus \& Blackman (2006) studied binary induced outflows. In their scheme a planet is not capable of unbinding the envelope, but could induce dynamo and poloidal flows.

\subsection{Planet dissipation inside the stellar envelope}

The structural changes that an AGB star undergoes in response to the dissipation of a planet in its interior are complex and have been extensively explored by Siess \& Livio (1999ab), and by Struck et al. (2002, 2004). In general, high accretion rates and the deposition of mass at the bottom of the convective envelope can produce substantial expansion of the star, even triggering Hot Bottom Burning at the base of the convective envelope.

An estimate of the maximum planet mass that can be evaporated inside an AGB envelope can be obtained by equating the location of the evaporation region (where the local sound speed in the stellar envelope matches the escape velocity from the planet's surface) to the energy required to expel the envelope. In Villaver \& Livio (2007) we show that planets with masses less than $15 M_{\mathrm{J}}$ (where $M_{\mathrm{J}}$ is the Jupiter mass) will evaporate inside the envelope of an AGB star with main sequence mass of $1 \mathrm{M}_{\odot}$. This mass limit is much higher, $\sim 120 M_{\mathrm{J}}$, if the planet (or brown dwarf) is engulfed inside the AGB envelope of a $5 \mathrm{M}_{\odot}$ star.

\subsection{Planets and horizontal branch stars}

A small fraction of stars lose their envelope during the Red Giant Branch and are left with with a very thin $\mathrm{H}$ envelope during then He-burning core phase, those are known as the hot sdB stars. The role of planets in the formation of these systems have been explored by Bear \& Soker (2011). A very interesting case along these lines is the planet found orbiting the Horizontal Branch star V391 Pegasi (Silvotti et al. 2007).

\section{The asymptotic giant branch}

Given the definition of $R_{*}^{\max }$ provided above, for all the initial orbits that satisfy the condition $r_{\mathrm{o}} \leqslant R_{*}^{\max }$ when the star expands during the AGB phase, the variation of the 
orbit can be approximated by $r(t)=r_{\mathrm{o}} M_{*} / M_{*}(t)$ where $r_{\mathrm{o}}$ is the initial orbital radius and $M_{*}(t)$ is time-dependent stellar mass caused by mass-loss (see Villaver \& Livio 2007). Given an initial orbital distance, if planet avoids entering the stellar envelope, its orbital radius at the end of the AGB evolution will be given by the initial-to final-mass relation. A planet orbiting a star with an initial mass of $5 \mathrm{M}_{\odot}$ will end as a white dwarf with mass $0.9 \mathrm{M}_{\odot}$ and thus the initial orbital distance of the planet will move a factor of 5.5.

\section{The planetary nebulae phase}

Until the PN phase the stellar effective temperature of the star has always remained lower than its main sequence value. However, once the star reaches the PN phase, irradiation effects are going to become important. The planet's orbit is not expected to change further at this stage. The stellar luminosity during this phase is of the order of $\times 10^{3} \mathrm{~L}_{\odot}$, and the stellar temperature can be as high as $380000 \mathrm{~K}$. The hydrogen ionizing photon flux, which is of the order of $10^{48} \mathrm{~s}^{-1}$ (Villaver et al. 2002b), is responsible for the PNe ionized line emission. The survival of a gas planet as the star evolves into the PN phase strongly depends on the planet's surface temperature, which ultimately determines whether or not high evaporation rates are set at the planet's surface.

Given the high effective temperature of the star, most of the stellar flux is emitted at short wavelengths, for which the material in the planet's atmosphere has a high optical depth. It can be shown that high exospheric temperatures are reached at the planet's upper atmosphere, and that it is expected that an outflow will develop as a consequence of the absorption of the XUV radiation (see Villaver \& Livio 2007). Since high temperatures can cause the outer layers of the planet to escape rapidly, in order to examine the planet survival, we have estimated the evaporation rate assuming hydrodynamic scape conditions. Those are shown in Figure 3, adapted from Villaver \& Livio (2007). The luminosities and effective temperatures of the central stars of PN evolve relatively quickly and the evaporation rates change accordingly.

\subsection{Planetary nebulae morphology}

The influence that a planet might have in the development of the PN morphology has been explore by Soker $(1996,1997,2001)$. In Soker (1997) the argument was given that common envelope interaction needed to account for $75 \%$ of all PN. Since there was not enough stellar companions to produce the large number of non-spherical PNe, a large number of planetary companions were needed. Recently de Marco \& Soker (2011) have revisited this earlier argument. By adopting a model whereby only $20 \%$ of all stars with masses between 1 and $8 \mathrm{M}_{\odot}$ make a $\mathrm{PN}$ they reducing the need for planetary shaping, and conclude that of the $80 \%$ of $1-8 \mathrm{M}_{\odot}$ stars that do not make a PN, about one-quarter do not even ascend the AGB due to interactions with stellar and substellar companions.

\section{White dwarfs}

A significant fraction of the white dwarf (WD) population shows traces of elements heavier than helium in their atmospheres. WDs have a high surface gravity and therefore, heavy elements are expected to sink in very short timescales leaving just pure $\mathrm{H}$ or $\mathrm{He}$ atmospheres. External sources must be responsible (i.e disrupted asteroids) for the presence of metals observed within cool WD photospheres, and circumstellar disks have been found in apparently single WDs polluted with metals (Gänsicke et al. 2008). Following this idea, Bonsor, Mustill \& Wyatt (2011), use a planetesimal belt whose inner edge is 


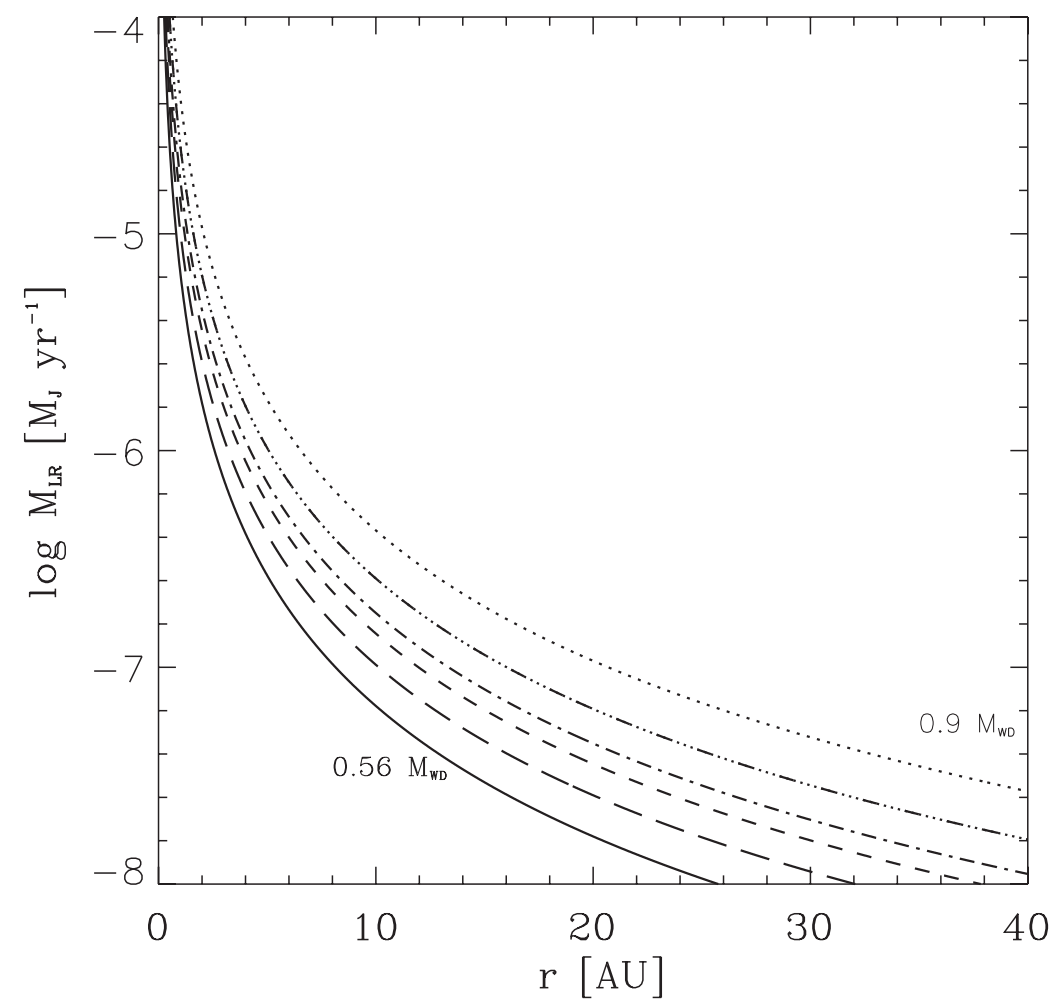

Figure 3. Evaporation rates (in logarithmic scale and $\mathrm{M}_{\mathrm{J}} \mathrm{yr}^{-1}$ ) versus orbital distance of a Jupiter-like planet under hydrodynamic escape conditions. The lines represent the radiation field for different central star masses $30000 \mathrm{yr}$ after the star enters the PN phase of evolution. The radiation (and therefore the evaporation rates) increases with the stellar mass.

truncated by a planet. A fraction of the mass scattered into the inner planetary system ends up tidally disrupted and accreted into the WD.

These disks are very different in nature from the one detected in the Hellix nebula (Su et al. 2007). In the Hellix the disk is probably the final evolution of the Solar System Kuiper/belt analogue (see e. g. Bonson \& Wyatt 2010).

\section{Summary}

It seems more than plausible that many planets may be accreted by their host stars. Although so far the results are based on a fairly limited sample, it appears that giant stars hosting planets show different properties to giant stars without planets. From the view point of stellar evolution, engulfment of a planet by a giant star it will most likely have an effect on the stellar structure and/or its subsequent evolution. In this review I have tried to summarize what are these expected effects.

\section{References}

Alexander, J. B. 1967, The Observatory, 87, 238

Bear, E. \& Soker, N. 2011, MNRAS, 411, 1792

Brown, J. A., Sneden, C., Lambert, D. L., \& Dutchover, E., Jr. 1989, ApJ Supplement Series, 71,293

Bonsor, A., Mustill, A. J., \& Wyatt, M. C. 2011, MNRAS, 414, 930 
Carlberg, J. K., Majewski, S. R., \& Arras, P. 2009, ApJ, 700, 832

Currie, T. 2009, ApJ, 694, L171

de Marco, O. \& Soker, N. 2011, PASP, 123, 402

de Medeiros, J. R., Da Rocha, C., \& Mayor, M. 1996, A\&A, 314, 499

Gänsicke, B. T., Koester, D., Marsh, T. R., Rebassa-Mansergas, A., \& Southworth, J. 2008, $M N R A S, 391, \mathrm{~L} 103$

Gray, D. F. 1981, ApJ, 251, 155

Iben, I., Jr. 1967, ApJ, 147, 624

Johnson, J. A., et al. 2007, ApJ, 670, 833

Johnson, J. A., Bowler, B. P., Howard, A. W., et al. 2010, ApJ Letters, 721, L153

Kumar, Y. B., Reddy, B. E., \& Lambert, D. L. 2011, ApJ, 730, L12

Livio, M. \& Soker, N. 2002, ApJ Letters, 571, L161

Lovis, C. \& Mayor, M. 2007, A\& $A, 472,657$

Nordhaus, J. \& Blackman, E. G. 2006, MNRAS, 370, 2004

Nordhaus, J., Blackman, E. G., \& Frank, A. 2007, MNRAS, 376, 599

Siess, L. \& Livio, M. 1999a, MNRAS, 304, 925

Siess, L. \& Livio, M. 1999b, MNRAS, 308, 1133

Silvotti, R., Schuh, S., Janulis, R., et al. 2007, Nature, 449, 189

Soker, N. 1996, MNRAS, 460, L53

Soker, N. 1997, ApJ Suplement Series, 112, 487

Soker, N. 2001, ApJ Letters, 324, 699

Struck, C., Cohanim, B. E., \& Willson, L. A. 2002, ApJ Letters, 572, L83

Struck, C., Cohanim, B. E., \& Willson, L. A. 2004, MNRAS, 347, 173

$\mathrm{Su}, \mathrm{K}$. Y. L., Chu, Y.-H., Rieke, G. H., et al. 2007, ApJ Letters, 657, L41

Villaver, E., García-Segura, G., \& Manchado, A., 2002a, ApJ, 571, 880.

Villaver, E., Manchado, A., \& García-Segura, G., 2002b, ApJ, 581, 1204.

Villaver, E. \& Livio, M., 2007, ApJ, 661, 1192.

Villaver, E. \& Livio, M., 2009, ApJ, 705, L81.

Wright, J. T., Upadhyay, S., Marcy, G. W., Fischer, D. A., Ford, E. B., \& Johnson, J. A. 2009, ApJ, 693, 1084 